\title{
A HIF1a-GPD1 feedforward loop inhibits the progression of renal clear cell carcinoma via mitochondrial function and lipid metabolism
}

Ren Liu ${ }^{1 \dagger}$, Yuanfa Feng ${ }^{2,3 \dagger}$, Yulin Deng ${ }^{1 \dagger}$, Zhihao Zou ${ }^{2}$, Jianheng Ye ${ }^{2}, Z_{\text {Zhiduan Cai }}^{4}$, Xuejin Zhu ${ }^{2,3}$, Yingke Liang ${ }^{2}$, Jianming Lư ${ }^{2}$, Hui Zhang ${ }^{2}$, Yong Luo ${ }^{5}$, Zhaodong Han², Yangjia Zhuo², Qingling Xie ${ }^{1,4}$, Chi Tin Hon 6 , Yuxiang Liang ${ }^{2^{*}}$, Chin-Lee $\mathrm{Wu}^{7,8^{*}}$ and Weide Zhong ${ }^{1,2,3,6,7,8^{*}}$ (D)

\begin{abstract}
Background: Hypoxia signaling, especially the hypoxia inducible factor (HIF) pathway, is a major player in clear cell renal cell carcinoma (ccRCC), which is characterized by disorders in lipid and glycogen metabolism. However, the interaction between hypoxia and lipid metabolism in ccRCC progression is still poorly understood.

Methods: We used bioinformatic analysis and discovered that glycerol-3-phosphate dehydrogenase 1 (GPD1) may play a key role in hypoxia and lipid metabolism pathways in cCRCC. Tissue microarray, IHC staining, and survival analysis were performed to evaluate clinical function. In vitro and in vivo assays showed the biological effects of GPD1 in ccRCC progression.

Results: We found that the expression of GPD1 was downregulated in CCRCC tissues, and overexpression of GPD1 inhibited the progression of $c \mathrm{RCC}$ both in vivo and in vitro. Furthermore, we demonstrated that hypoxia inducible factor-1a (HIF1a) directly regulates GPD1 at the transcriptional level, which leads to the inhibition of mitochondrial function and lipid metabolism. Additionally, GPD1 was shown to inhibit prolyl hydroxylase 3 (PHD3), which blocks prolyl-hydroxylation of HIF1a and subsequent proteasomal degradation, and thus reinforces the inhibition of mitochondrial function and phosphorylation of AMPK via suppressing glycerol-3-phosphate dehydrogenase 2 (GPD2).
\end{abstract}

\footnotetext{
*Correspondence: doctorbaby@126.com; cwu2@mgh.harvard.edu; zhongwd2009@live.cn

tRen Liu, Yuanfa Feng and Yulin Deng contributed equally to this work. 2Department of Urology, Guangdong Key Laboratory of Clinical Molecular Medicine and Diagnostics, Guangzhou First People's Hospital, School of Medicine, South China University of Technology, Guangzhou 510180, China ${ }^{7}$ Departments of Urology, Massachusetts General Hospital and Harvard Medical School, Boston, MA 02114, USA

'Guangdong Provincial Institute of Nephrology, Nanfang Hospital, Southern Medical University, Guangzhou 510515, China

Full list of author information is available at the end of the article
}

(c) The Author(s). 2021 Open Access This article is licensed under a Creative Commons Attribution 4.0 International License, which permits use, sharing, adaptation, distribution and reproduction in any medium or format, as long as you give appropriate credit to the original author(s) and the source, provide a link to the Creative Commons licence, and indicate if changes were made. The images or other third party material in this article are included in the article's Creative Commons licence, unless indicated otherwise in a credit line to the material. If material is not included in the article's Creative Commons licence and your intended use is not permitted by statutory regulation or exceeds the permitted use, you will need to obtain permission directly from the copyright holder. To view a copy of this licence, visit http://creativecommons.org/licenses/by/4.0/ The Creative Commons Public Domain Dedication waiver (http://creativecommons.org/publicdomain/zero/1.0/) applies to the data made available in this article, unless otherwise stated in a credit line to the data. 
Conclusions: This study not only demonstrated that HIF1a-GPD1 forms a positive feedforward loop inhibiting mitochondrial function and lipid metabolism in $\mathrm{CCRCC}$, but also discovered a new mechanism for the molecular basis of HIF1a to inhibit tumor activity, thus providing novel insights into hypoxia-lipid-mediated ccRCC therapy.

Keywords: Hypoxia, ccRCC, Metabolism, HIF1a, GPD1

\section{Background}

Renal cancer is a common urologic cancer, and the pathological classification is diverse. It consists mainly of renal clear cell carcinoma, most of which occurs in renal tubular epithelial cells [1]. Clear cell renal cell carcinoma (ccRCC) is histologically defined as malignant renal tubular epithelial cells with transparent and bright cytoplasm due to the massive accumulation of lipids and glycogen [2], suggesting that altered lipid and glucose metabolism plays a major role in its progression. From an epigenetic point of view, the main feature of ccRCC is the biallelic loss of the von Hipper Lindau (VHL) tumor suppressor. VHL encodes an E3 ubiquitin ligase that degrades hypoxia inducible factors (HIF) $1 \alpha$ and HIF2 $\alpha$ [3], which leads to deregulation of the hypoxia pathway. In contrast to many tumor types, HIF1 $\alpha$ and HIF2 $\alpha$ have opposite roles in ccRCC biology, where HIF $1 \alpha$ acts as a tumor suppressor and HIF $2 \alpha$ acts as an oncogene [4]. This has promoted the development of HIF2 $\alpha$-specific inhibitors, which exhibit exceptional targeting effects in ccRCC xenograft models and show efficacy in a subset of patient-derived xenograft models and clinical responses in some phase I clinical trials [5]. While there are many studies focusing on the mechanism underlying the HIF $2 \alpha$-dependent promotion of ccRCC progression, studies regarding the mechanism of how HIF1 $\alpha$ inhibits tumors are insufficient. Thus far, only one study by Florinda et al. has shown that HIF1 $\alpha$ functions as an inhibitor of aspartate biogenesis by repressing glutamine oxidation and reductive carboxylation pathways via negatively regulating two key players, cytosolic glutamic-oxaloacetic transaminase-1 (GOT1) and mitochondrial GOT2 [6].

Proline hydroxylase (PHD) enzymes catalyze the oxygen-dependent hydroxylation of specific proline residues of HIFs, thereby causing binding to pVHL, ubiquitination, and subsequent proteasomal degradation. Under hypoxic conditions, the PHD enzymes are inhibited. As a result, HIF- $\alpha$ escapes degradation and forms a dimer with the HIF-1 $\beta$ subtype, which is expressed constitutively. This complex is then transported into the nucleus and binds to the hypoxia response element (HRE) within the promoter region upstream of the coding region, thereby affecting the transcription of a variety of genes, which also leads to lipid reprogramming and other metabolic disorders [7]. Lipidomic studies have demonstrated that fatty acids, triglycerides, and cholesterol exist at higher levels in ccRCC tissues as compared with normal kidney tissues [8]. Therefore, hypoxia-lipid-pathways play a significant role in the progression of ccRCC, but the underlying mechanism is not clear.

In this study, we analyzed and screened the RNA-seq data of 530 ccRCC patients in The Cancer Genome Atlas (TCGA) from two aspects, hypoxia and lipid metabolism, using bioinformatic analysis. We found that GPD1 was significantly involved in hypoxia and lipid metabolism pathways in the progression of ccRCC. Glycerol-3-phosphate dehydrogenase 1 (GPD1), catalyzes the reaction of acetone dihydrogen phosphate (DHAP) and nicotinamide adenine dinucleotide (NADH) into glycerol-3-phosphate (G3P) and nicotinamide adenine dinucleotide (NAD+), which provides substrates for the synthesis of glycerol, and links lipogenesis and carbohydrate metabolism [9]. GPD1 and GPD2 isoenzymes together form the glycerol triphosphate shuttle system. GPD1 functions in the cytoplasm, and GPD2 catalyzes the reversible conversion in the mitochondria and transfers electrons to the oxidative respiratory chain to promote aerobic phosphorylation [10, 11]. Studies have shown that knocking down the expression of GPD2 can induce AMPK phosphorylation [12] and inhibit mitochondrial functioning [13], which results in a reduction of energy supply and the suppression of tumors. Our previous study also found that the overexpression of GPD1 in prostate cancer and lung cancer cells inhibits the aerobic phosphorylation of mitochondria, which may be related to the deregulation of the hypoxia pathway [14]. Another study reported that GPD1 was minimally expressed in ccRCC cancer tissues via proteomics [15], but there is a lack of in-depth research on the specific functions and mechanisms.

\section{Materials and methods}

\section{Patients and tissues}

This study was approved by the Human Ethics Committee of the Public Health Department of the People's Republic of China and the Massachusetts General Hospital. All patients signed an informed consent form. According to relevant laws and legal standards, all samples were processed anonymously.

The 17 pairs of matched frozen samples of renal cell carcinoma and benign renal tissue adjacent to the cancer, as well as the human ccRCC tissue microarrays 
(TMAs) used in this study, which contained 69 case samples, were all from the Massachusetts General Hospital. The inclusion criterion was that the patient did not receive chemotherapy or radiotherapy before surgery. Each case was diagnosed and graded by two pathologists separately and re-examined by HE staining. Relevant clinical information, including age, gender, Fuhrman grade, AJCC (American Joint Committee on Cancer) tumor $\mathrm{T}$ stage, tumor size, metastasis status, and overall survival time, was collected. The overall survival was defined as the time from the date of surgery to the last follow-up or death.

\section{Cell culture}

All human cell lines were purchased from ATCC (American Type Culture Collection, Manassas, Virginia, USA), including HK2, RCC4, 769P, ACHN, Caki1, and Caki2. All cell lines were authenticated by polymorphic short tandem repeat profiling and were cultured at $37^{\circ} \mathrm{C}$ in a $5 \% \mathrm{CO}_{2}$ incubator with appropriate cell culture medium according to ATCC guidelines (supplementary materials). Hypoxia cell culture was achieved in a hypoxia chamber flushed with a pre-analyzed gas mixture of $1 \% \mathrm{O}_{2}, 5 \% \mathrm{CO}_{2}$, and $95 \% \mathrm{~N}_{2}$ at $37^{\circ} \mathrm{C}$ for $24 \mathrm{~h}$.

\section{Construction and transfection of cell lines}

To construct the lentiviral plasmids, an encoding vesicular stomatitis virus was used to envelope the protein plasmid pMD.2G $(5 \mu \mathrm{g})$ and packaging plasmid psPAX2 $(10 \mu \mathrm{g})$ by using the calcium phosphate method. Then, HEK293 cells were transfected with the lentiviral plasmids $(12 \mu \mathrm{g})$, and the supernatant virus solution was collected after $48 \mathrm{~h}$. Caki1 and 769P cells were seeded in 12-well plates and transfected with the supernatant virus solution for $12 \mathrm{~h}$ and incubated for another $24 \mathrm{~h}$ with fresh medium. Via flow cytometric analysis, we observed the expression of GFP to confirm the transfection. We collected the expanded cultured tumor cells, performed flow sorting, and enriched GFP + tumor cells through multiple sortings, such that GFP+ cells were more than 99\%, and the expression was stable. Through western blotting, we identified the Caki1 and 769P cell lines that stably overexpressed GPD1.

Human GPD1-specific siRNA, human GPD2-specific siRNA, human HIF1 $\alpha$-specific siRNA, human HIF2 $\alpha$ specific siRNA, and negative control siRNA were purchased from GenePharma (Suzhou, China). The siRNA sequences are shown in the supplementary materials. Western blotting analyses were conducted $48 \mathrm{~h}$ after transfection to test the transfection efficacy at the protein level.

\section{qRT-PCR}

Quantitative analysis of mRNA expression was performed using qRT-PCR according to our published protocols [14, 16]. Sequences of all primers used for PCR are provided in the supplementary materials.

\section{Western blot analysis}

Quantitative analysis of protein expression in cell lines and clinical tissues was performed using western blotting according to the protocol of our previous studies [14, $16]$. A list of antibodies is provided in the supplementary materials.

\section{Animals and xenografts}

All animal experiments were performed in accordance with the guidelines of the Institute for Laboratory Animal Research and the National Cancer Center Research Institute. Four-to-five-week-old BALB/c nude male mice were purchased from the Experimental Animal Center of Sun Yat-sen University (Guangzhou, China). For each xenograft assay, $5 \times 10^{6}$ cells were subcutaneously injected into the flanks of nude mice. Tumor sizes and body weights were measured every three days. Tumor volumes were determined by the following formula: $(\mathrm{L} \times$ $\left.\mathrm{W}^{2}\right) / 2$.

\section{Immunohistochemistry}

GPD1 protein expressions in ccRCC tissues and normal kidney tissues were detected by immunohistochemistry (IHC) according to our previous published protocols $[14,16]$. The GPD1 antibody was purchased from Santa Cruz (sc-376,219, Dallas, Texas, USA).

\section{Cell proliferation and migration analyses}

Cell proliferation was detected by a CCK-8 (Cell Counting Kit-8, MA0218, Meilunbio, Dalian, China) assay. Cell migration was detected by a wound healing assay as previously described $[14,16]$.

\section{Cell cycle and apoptosis analyses}

Cell cycle analysis was performed using the Cell Cycle Staining kit (70-CCS012, Multi Sciences, Hangzhou, China) according to the manufacturer's protocol. For the cell apoptosis analysis, the Annexin V-FITC/PI Apoptosis Detection Kit (70-AP101-100, Multi Sciences) was used according to the manufacturer's instructions. The stained cells were washed with PBS and analyzed via flow cytometry on a CytoFLEX system (CytoFLEX S, Beckman Coulter, Brea, California, USA).

\section{Seahorse assay}

Cells were seeded $\left(8 \times 10^{3}\right.$ cells/well $)$ in an $\mathrm{XFe} 24$ cell culture microplate $(100,777$, Agilent, Santa Clara, California, USA). We used the XFe24 Cell Mito Stress Test (100,850, Agilent) to determine mitochondrial function by directly measuring the oxygen consumption rate (OCR) of each cell line as described in our previous 
studies [14, 16]. The XF ATP Rate Assay (103,592, Agilent) was used to simultaneously measure the rate of ATP production and was run in an XFe24 Extracellular Flux Analyzer (Agilent). The data were analyzed using the Wave software program 2.6.0 (Agilent).

\section{Immunofluorescence}

Cells were seeded in 6-well plates, treated with $4 \%$ formaldehyde for $15 \mathrm{~min}$, and then homogenized in $0.5 \%$ Triton X-100 for $15 \mathrm{~min}$. Next, the primary antibodies, GPD1 (sc-376,219, Santa Cruz) and HIF1 $\alpha$ (20960-1AP, Proteintech, Wuhan, China), were diluted in 5\% BSA respectively and incubated with the cells overnight at $4{ }^{\circ} \mathrm{C}$. After that, the cells were washed three times with PBS and then incubated with a second antibody (Alexa Fluor, Boster, Guangzhou, China) at $25^{\circ} \mathrm{C}$ for $1 \mathrm{~h}$. Finally, a DAPI antibody was incubated with the cells for $5 \mathrm{~min}$, followed by washing three times and the addition of the fluorescence quenching agent. The cells were imaged using a confocal laser scanning microscope (LSM880, Leica, Germany).

\section{CUT\&RUN}

CUT\&RUN experiments were conducted using 300,000 RCC4 cells with the CUT\&RUN assay kit (\#86652, Cell Signaling Technology, Beverly, MA, USA). In brief, cells were washed and bound to concanavalin A-coated magnetic beads, and then performed permeabilization was performed with Wash Buffer containing 0.05\% digitonin. After that, cells were incubated with $5 \mu \mathrm{g}$ of HIF1 $\alpha$ antibody (ab1, Abcam, Shanghai, China) for $8 \mathrm{~h}$ at $4{ }^{\circ} \mathrm{C}$. Then, cell-bead slurry was washed twice with Dig Wash Buffer containing $0.05 \%$ digitonin and incubated with Protein A-MNase for $1 \mathrm{~h}$ at $4{ }^{\circ} \mathrm{C}$. After washing with Wash Buffer containing $0.05 \%$ digitonin, $2 \mathrm{mM} \mathrm{CaCl}_{2}$ was added into the cell-bead slurry to initiate Protein AMNase digestion, which was then incubated for $30 \mathrm{~min}$ on ice. After the antibody-specific incubation period, the reaction was stopped with the addition of one volume of 2x Stop Buffer containing $20 \mathrm{mM}$ EDTA, 0.05\% digitonin, $5 \mathrm{mg} / \mathrm{ml} \mathrm{RNase} \mathrm{A,} \mathrm{and} 2 \mathrm{pg} / \mathrm{ml}$ heterologous spikein DNA. CUT\&RUN fragments were released by incubation for $30 \mathrm{~min}$ at $37^{\circ} \mathrm{C}$ followed by centrifugation for 5 $\min$ at $16,000 \times g$. After centrifugation, the supernatant was recovered, and the DNA extracted using DNA Purification Buffers and Spin Columns (\#14209, Cell Signaling Technology). The resulting DNA products were quantified by qRT-PCR as described above. The primers used for qRT-PCR amplification are described in the supplementary materials.

\section{Luciferase reporter assay}

The HIF $1 \alpha$ plasmid, firefly luciferase plasmid GPD1HBS promoter, firefly luciferase plasmid GPD2-HBS1 plus HBS2 promoter, and firefly luciferase plasmid GPD2-HBS2 promoter were all purchased from GenePharma (Suzhou, China) along with the renilla luciferase plasmid. HEK293 cells were grown to a density of 5000 cells per well in a 96-well plate and transfected with the renilla luciferase plasmid, HIF1 $\alpha$ plasmid, and firefly luciferase plasmid with the indicated promoter region. After $48 \mathrm{~h}$, luciferase activity was measured by using the Firefly \& Renilla Luciferase Reporter Assay Kit (MA0518, Meilunbio, Dalian, China) and a Varioskan Full Wavelength Fluorescence Microplate Reader (Thermo Fisher Scientific, Shanghai, China). The relative luciferase activity was calculated from the firefly luciferase normalized to renilla luciferase. The luciferase reporter assays were conducted in triplicate, and repeated independently for three times. The promoter sequences are listed in the supplementary materials.

\section{Coimmunoprecipitation (Co-IP)}

HEK293 cells were grown to 70\% confluence in 6-well plates and then transfected with GPD1 or negative control plasmids for $24 \mathrm{~h}$. Cell lysates were prepared for CoIP analysis (\#88805, Thermo Fisher Scientific). Briefly, beads were prewashed two times with $1 \times$ modified coupling buffer and then incubated with $5 \mu \mathrm{g}$ of HIF1 $\alpha$ antibody (ab8366, Abcam) for $15 \mathrm{~min}$. After that, the beads were washed three times with $1 \times$ modified coupling buffer, and the antibody was crosslinked in $1 \mathrm{ml}$ of disuccinimidyl suberate solution for $30 \mathrm{~min}$. Then, the beads were washed three times with elution buffer, followed by two IP lysis/wash buffer washes. After that, the cell lysates were incubated with the beads for $8 \mathrm{~h}$ at $4{ }^{\circ} \mathrm{C}$. The next day, the beads were washed twice with $1 \mathrm{ml}$ of IP lysis/wash buffer, followed by a final wash with ultrapure water. The bound antigen was finally eluted and prepared for western blotting analysis.

\section{Oil red $O$ staining}

Cells were plated in six-well plates in triplicate, followed by rinsing with PBS twice. Then, the cells were fixed with $10 \%$ formaldehyde for $30 \mathrm{~min}$. After that, $1 \mathrm{ml}$ of $60 \%$ isopropanol per well was used to wash the cells, and $1 \mathrm{ml}$ of $0.5 \%$ Oil Red O solution (MA0120, Meilunbio, Dalian, China) was incubated with the cells for $10 \mathrm{~min}$, after which the cells were washed five times with water. The nuclei were stained with hematoxylin staining solution (MB9897, Meilunbio) and differentiated with $1 \%$ hydrochloric acid solution for $1 \mathrm{~min}$. Filter paper was used to absorb the surrounding moisture, and then the slides were mounted with glycerin gelatin and observed under a microscope. 


\section{Free fatty acid quantitation assay}

A Free Fatty Acid Quantitation Kit was used (MAK044, Merck, Darmstadt, Germany) to quantify the free fatty acid levels. According to the protocol, cells $\left(1 \times 10^{6}\right)$ were homogenized in $200 \mu \mathrm{l}$ of $1 \%(\mathrm{w} / \mathrm{v})$ Triton X-100 in chloroform. After centrifugation at $13,000 \times g$ for $10 \mathrm{~min}$, samples in the lower organic phase were collected, air dried at $50{ }^{\circ} \mathrm{C}$ to remove chloroform, and then placed under a vacuum for $30 \mathrm{~min}$ to remove any organic solvent. The dried samples were then dissolved in $200 \mu \mathrm{l}$ of fatty acid assay buffer by sonication and vortexed until the samples were homogeneous. Then, according to the manufacturer's protocol, a reaction mix containing $2 \mu \mathrm{l}$ ACS reagent, $44 \mu \mathrm{l}$ fatty acid assay buffer, $2 \mu \mathrm{l}$ fatty acid probe, $2 \mu \mathrm{l}$ enzyme mix, and $2 \mu \mathrm{l}$ enhancer was set up and incubated with samples for $30 \mathrm{~min}$ at $37^{\circ} \mathrm{C}$. Free fatty acid levels were then determined by colorimetric absorbance at $570 \mathrm{~nm}$.

\section{Bioinformatic analysis}

Data from 539 ccRCC patients and 72 paired kidney tissues were obtained from the TCGA database (The Cancer Genome Atlas, https://portal.gdc.cancer.gov). To detect the hypoxia- and lipid-related subtypes in TCGAKIRC patients, dimensionality reduction and clustering was performed using the 'Rtsne' package and 'kmeans' function based on the hallmark gene sets of hypoxia- or lipid-related genes, respectively. The gene sets of 'HALL MARK_HYPOXIA' and 'HALLMARK_FATTY_ACID_ METABOLISM' were obtained from the Molecular Signatures Database (MSigDB version 6.0). Kaplan-Meier analysis and the log-rank test were used to compare the overall survival outcomes in different clusters using the $\mathrm{R}$ package "survival". Then, the hypoxia- and lipidrelated differentially expressed genes (DEGs) were obtained by the expression comparison between different prognostic clusters. The intersection of hypoxia-related DEGs and lipid-related DEGs was defined as 'hypoxia/ lipid DEGs'. To further narrow down the significant features among hypoxia/lipid DEGs, the Least Absolute Shrinkage and Selection Operator (LASSO) method was further performed based on the R package 'glmnet' [17]. With the optimal lambda value, the candidate hypoxia/ lipid DEGs were selected for further analysis. In addition, a hypoxia/lipid-related signature was constructed based on the selected candidate hypoxia/lipid DEGs. Risk stratification analysis, i.e. Kaplan-Meier analysis, Wilcoxon signed-rank test, and the timedependent receiver operating characteristic (ROC) curve, were utilized to evaluate the predictive value of the hypoxia/lipid-related signature. All of the above bioinformatic analyses were performed in $\mathrm{R}$ (version 4.0.2).

The RNA-seq dataset (GSE146982, https: //www.ncbi. nlm.nih.gov/geo/) was analyzed using the GSEA (Gene
Set Enrichment Analysis) software program ((http:// www.broadinstitute.org/gsea).

\section{Statistical analysis}

SPSS software (SPSS 23.0, SPSS Inc.) and GraphPad Prism 7 (https://www.graphpad.com/) were used for statistical analysis. Comparisons were performed by using a paired Student's $t$ test or variance analysis (ANOVA). Kaplan-Meier survival curves were used to assess survival time, and log-rank tests were used for comparison. Cox regression was performed for univariate and multivariate analyses. A $p<0.05$ indicated that the difference was statistically significant.

\section{Results}

GPD1 serves an important role in hypoxia and lipid metabolism pathways in cCRCC

The discovery cohort contained 530 ccRCC patients from the TCGA database, including the corresponding RNA-seq results and clinical information. Based on the expression matrix of 200 hypoxia hallmark genes as defined by the Broad Institute Molecular Signatures database, a non-linear dimensionality reduction algorithm tSNE was used to divide all patients into three groups (Groups 1, 2, and 3). A comparison of survival curves showed obvious significant differences among the three groups (log-rank test, $p<0.001$ ) (Fig. 1A). The overall survival rates of the patients in Group 1 were significantly higher than those of the patients in Group 3, which indicated that the hypoxia status was lowest in Group 1 and highest in Group 3. We further divided all patients into three lipid-related groups using the same method based on the expression matrix of 200 lipid metabolism hallmark gene sets. Similarly, Group 1, with the best overall survival, was the low-lipid metabolite group, while Group 3, with the worst overall survival, was the high-lipid metabolite group (Fig. 1B). Through differential gene analysis $(|\log 2 \mathrm{FC}|>1$, FDR-adjusted $p<0.01)$, 383 genes were found to be differentially expressed in the hypoxia ${ }^{\text {high }}$ (Group 3) and hypoxia ${ }^{\text {low }}$ (Group 1) groups, and there were 720 DEGs between the lipid metabolism $^{\text {high }}$ (Group 3) and lipid metabolism ${ }^{\text {low }}$ (Group 1) groups (Fig. 1C). Next, we obtained 82 genes by overlapping the hypoxia-related DEGs, the lipidrelated DEGs, and the DEGs between the ccRCC and benign tissues acquired from TCGA $(|\log 2 \mathrm{FC}|>1$, FDRadjusted $\mathrm{p}<0.01$ ) (Fig. 1D).

A LASSO Cox regression model was used to identify the most significant prognostic gene signature from these 82 hypoxia-lipid-related DEGs. The optimal gene signature consisted of 24 prognostic DEGs, and the corresponding coefficients were calculated (Fig. 1E and $\mathrm{S} 1 \mathrm{~A})$. The ROC curve analysis suggested that the prognostic accuracy for these 24 genes was 0.8 (Fig. S1B). 


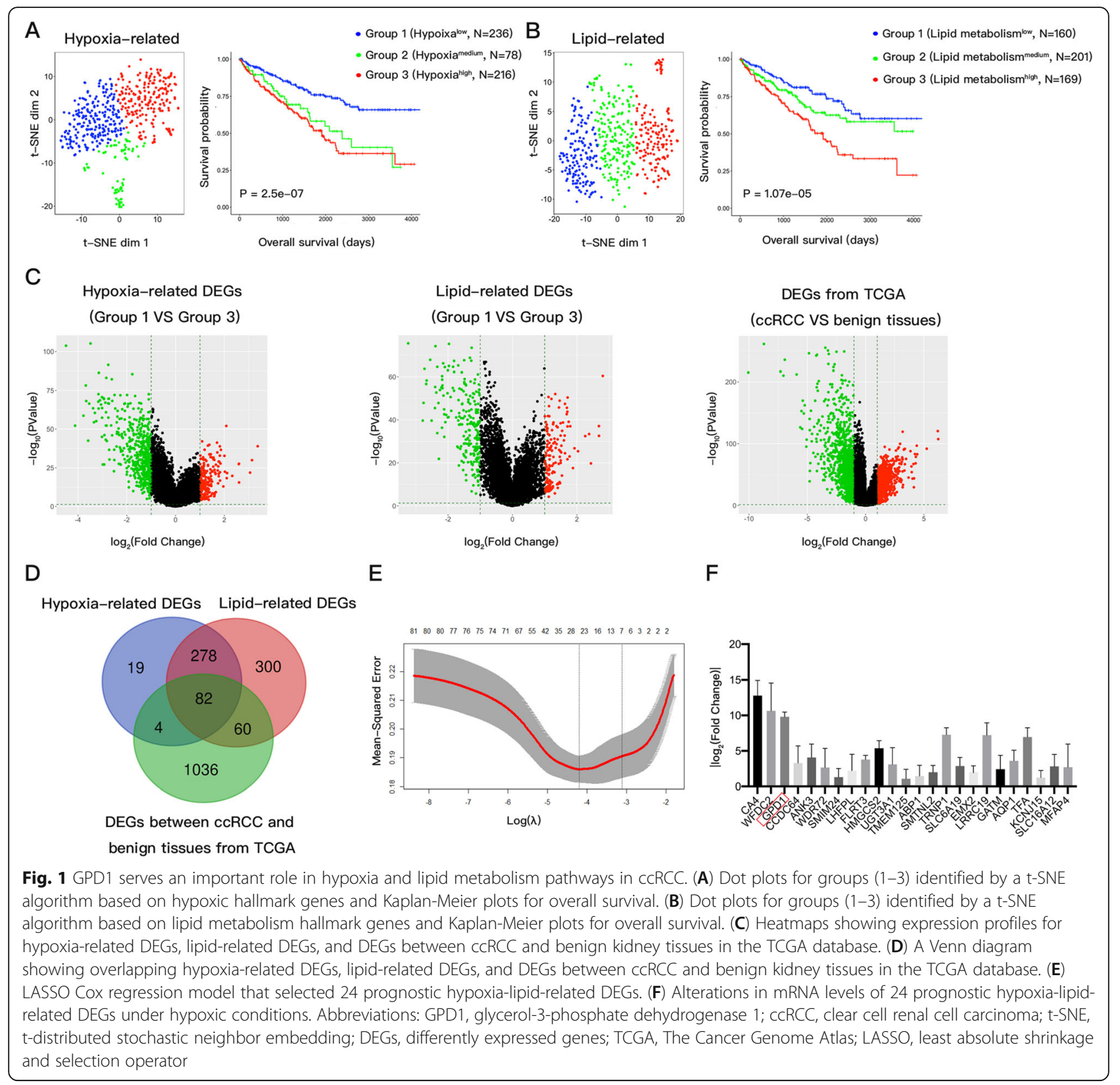

The distribution of risk score showed that patients who died had higher risk scores than patients who lived $(p<0.05)$. Using this 24-gene formula, the risk score of each patient in the TCGA cohort was calculated. The patients were then dichotomized equally into a high-risk group and a low-risk group according to the median risk score (Fig. S1C). KaplanMeier analysis showed that patients in the high-risk group had worse outcomes than those in the low-risk group (HR = 2.67, 95\% CI 1.96 to $3.62, p=1.20 \mathrm{e}-05$ ) (Fig. S1D).

We found that 9 out of these 24 genes were previously studied in ccRCC [18-26]. We then cultured the ccRCC cell line 769P under normoxic and hypoxic conditions and assessed the expressions of the 24 genes, which were
CA4, WFDC2, GPD1, CCDC64, ANK3, WDR72, SMIM24, LHFPL, FLRT3, HMGCS2, UGT3A1, TMEM125, ABP1, SMTNL2, TRNP1, SLC6A19, EMX2, LRRC19, GATM, AQP1, TFA, KCNJ15, SLC16A12 and MFAP4. We found that the mRNA expression of GPD1 was the one of the most significantly altered under hypoxic conditions (Fig. 1F).

The expression of GPD1 was downregulated in CCRCC tissues and inhibited the progression of CCRCC in vitro and in vivo

To investigate the expression and clinical significance of GPD1, we found that GPD1 mRNA was expressed less 
in ccRCC tissues as compared to normal kidney tissues based on the TCGA-KIRC database $(p<0.01)$ (Fig. S2A). The protein expression of GPD1 in ccRCC and normal tissues was examined by IHC and western blotting. We found that GPD1 protein was mainly expressed in the cytoplasm of renal tubular epithelial cells, and the expression level in benign tissues was much higher than that in tumor tissues (Fig. 2A and S2B).

The associations between GPD1 expression and the clinical pathological characteristics of ccRCC patients based on our TMA data are shown in Table 1 (Fig. 2B). The expression of GPD1 was significantly associated with vital status (alive) $(p=0.039)$; however, there was no significant association between GPD1 expression and tumor stage, Fuhrman grade, or tumor size. A survival comparison showed that high GPD1 protein expression correlated with significantly better overall survival (logrank test, $p=0.023$ ) (Fig. 2C). Further univariate and multivariate Cox regression analyses revealed that GPD1 was an independent predictor of overall survival, which indicated that GPD1 is a favorable prognostic indicator (Table 2).

We examined the expression of GPD1 in renal tubular epithelial cells (HK2) and various renal ccRCC cell lines and found that GPD1 was generally expressed at a lower level in tumor cell lines (Fig. S2C). Therefore, we established the GPD1overexpressing stable ccRCC cell lines, Cakil and 769P, via lentivector transduction and examined the expression via western blotting and qRT-PCR analyses (Fig. S2D). A CCK-8 assay showed that overexpression of GPD1 inhibited cell proliferation in both Caki1 and 769P cells (Fig. 2D). The colony formation abilities of GPD1-overexpressing ccRCC cells were greatly attenuated compared with the negative control groups (Fig. 2E and S2E). The wound healing and Transwell assays indicated that overexpression of GPD1 protein suppressed the migration and invasion of Caki1 and 769P cells (Fig. 2F, G and S2F). The cell cycle assay indicated that overexpression of GPD1 protein increased the percentage of G0/G1 phase cells (Fig. 2H). Moreover, we also performed flow cytometry analysis to determine the effect of GPD1 on apoptosis. The results showed that GPD1 significantly promoted apoptosis in Caki1 and 769P cells (Fig. 2I). Based on these results, we were interested in the role of GPD1 in tumor growth in vivo. As such, we tested the effect of Caki1 and 769P cell lines that stably expressed GPD1 in a murine xenograft model on tumor growth as compared to vector control cell lines. As shown in Fig. 2J, tumor growth was significantly inhibited by the overexpression of GPD1, and the tumors in the GPD1-overexpressing groups also had smaller sizes and lower weights than those in the control groups (Fig. S2G).
Overexpression of GPD1 inhibited mitochondrial function and induced AMPK phosphorylation by suppressing the expression of GPD2

We performed the seahorse assay for Cakil and 769P cells with GPD1 overexpression to detect the OCR. The results showed that overexpression of GPD1 can significantly inhibited the levels of baseline respiration, ATP production, and spare respiratory capacity (Fig. 3A), which were consistent with the results after knocking down the expression of GPD2 (Fig. S3A and S3B). Western blotting analysis and qRT-PCR indicated that the protein and mRNA expression levels of GPD2 were significantly inhibited by the overexpression of GPD1. Additionally, AMPK phosphorylation was obviously induced, and the tumor mTOR pathway was suppressed (Fig. 3B and S3C). These results are consistent with the knockout of GPD2 in previous studies [12]. Moreover, the expressions of PCNA (a proliferation marker), Bcl-2 (an apoptosis marker), and MMP-2 (an invasion marker) were both downregulated in ccRCC cells with GPD1 overexpression.

Therefore, we hypothesized that GPD1 may inhibit mitochondrial function, which affected the energy supply, by inhibiting the expression of GPD2 and then inducing AMPK phosphorylation, which could suppress the progression of ccRCC. To confirm this, we rescued the expression of GPD2 in Caki1 and 769P cells with the overexpression of GPD1. Rescue of GPD2 expression yielded a significantly weak phosphorylated AMPK signal (Fig. 3C) and restoration of mitochondrial function (Fig. 3D). Furthermore, restoration of GPD2 in Caki1GPD1 and 769P-GPD1 cells significantly recovered their proliferation, migration, and invasion abilities (Fig. 3E, F, and G). However, the underlying regulatory mechanism is still unclear, and further research is needed.

\section{HIF1a directly targeted and positively regulated GPD1 and negatively regulated GPD2 at the transcriptional level}

Based on the above results, we hypothesized that GPD1 influenced the expression of GPD2 through the hypoxia pathway. To uncover the role of GPD1 and GPD2 under hypoxic conditions, HK2 cells and various renal ccRCC cell lines were exposed to hypoxia for $24 \mathrm{~h}\left(1 \% \mathrm{O}_{2}\right)$. We observed that the protein expression of GPD1 was significantly upregulated, while the protein expression of GPD2 was reduced (Fig. 4A). The mRNA expression of both GPD1 and GPD2 was similar to the protein expression (Fig. S4A). These observations indicated that GPD1 and GPD2 expression might be transcriptionally regulated after hypoxia exposure. To examine the possibility that GPD1 and GPD2 expressions were regulated by HIF transcription factors, we treated the ccRCC cell line RCC4 with a PHD inhibitor, dimethyloxalylglycine 


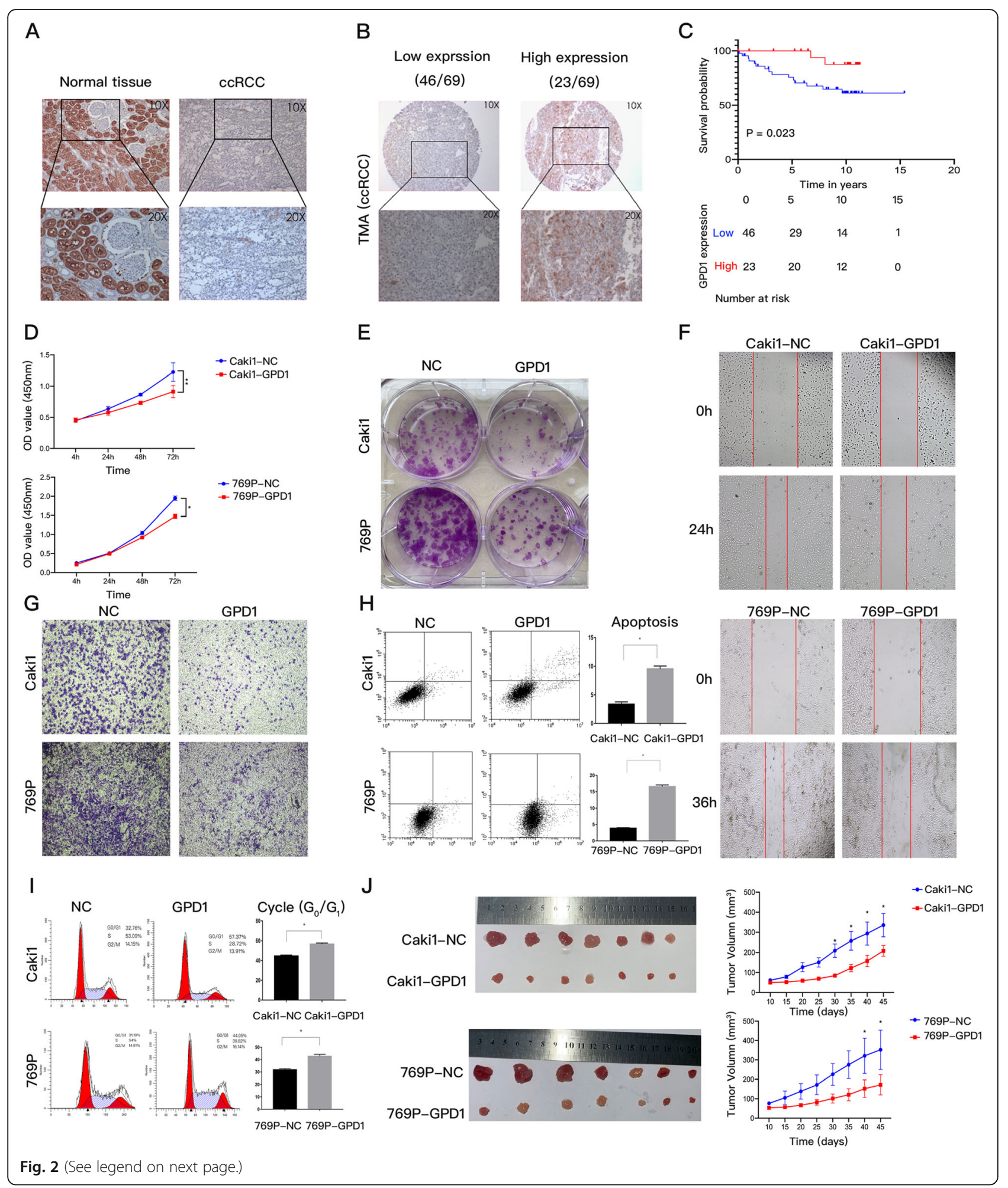


(See figure on previous page.)

Fig. 2 The expression of GPD1 was downregulated in ccRCC tissues and inhibited the progression of ccRCC in vitro and in vivo. (A) Immunostainings of GPD1 protein in ccRCC tissues and paired normal kidney tissues. (B) Immunostainings of GPD1 protein in 69 ccRCC samples of TMA. (C) Kaplan-Meier curves for overall survival between the GPD1 high-expression group and the GPD1 low-expression group based on GPD1 IHC scores (8-12, GPD1 high-expression, $n=23$ vs. 0-6, GPD1 low-expression, $n=46)$. (D-E) Cell proliferation as determined by CCK-8 and colony formation assays. (F-G) Cell migration and invasion as determined by wound healing and Transwell assays. Red lines denote the margins of the wound. (H) Cell apoptosis analysis of Caki1 and 769P cells overexpressing GPD1 or a negative control. (I) Cell cycle analysis of Caki1 and 769P cells overexpressing GPD1 or a negative control. The results are presented as the mean + SD of three independent experiments. (J) Images of tumors from the subcutaneous xenograft models implanted using Caki1 and 769P cells that stably overexpressed GPD1 or a negative control. The growth curves of tumors are shown $\left({ }^{*} p<0.05,{ }^{* *} p<0.01\right)$. Abbreviations: GPD1, glycerol-3-phosphate dehydrogenase 1; $c$ CRCC, clear cell renal cell carcinoma; TMA, tissue microarray; IHC, immunohistochemistry; TCGA, the cancer genome atlas; CCK-8, cell counting kit

(DMOG), and a proteasome inhibitor, MG132, both of which stabilize HIFs. As shown in Fig. 4B, both DMOG and MG132 increased the expression of HIF1 $\alpha$ and HIF2 $\alpha$, which might lead to the overexpression of GPD1 and inhibition of GPD2. Next, GPD1 expression was assessed in the ccRCC cell line RCC4 after siRNA-mediated knockdown of HIF1 $\alpha$ and HIF $2 \alpha$. si-HIF1 $\alpha$ significantly reduced the expression of GPD1 and increased the expression of GPD2, while si-HIF2 $\alpha$ did not play any role (Fig. 4C).

To further validate whether HIF1 $\alpha$ directly binds to the GPD1 and GPD2 promoter, the JASPAR database was analyzed for HIF-binding sites (HBS) within the GPD1 and

Table 1 Association between expression of GPD1 and clinical characteristics in CCRCC patients

\begin{tabular}{|c|c|c|c|}
\hline Characteristic & GPD1 & & $P$-value \\
\hline & Low $(n=46)$ & $\operatorname{High}(n=23)$ & \\
\hline Age (years) & & & 0.097 \\
\hline$<=65$ & 32 & 20 & \\
\hline$>65$ & 14 & 3 & \\
\hline Sex & & & 0.832 \\
\hline Female & 9 & 5 & \\
\hline Male & 37 & 18 & \\
\hline Fuhrman grade & & & 0.302 \\
\hline $1 / 2$ & 29 & 11 & \\
\hline $3 / 4$ & 17 & 12 & \\
\hline Tumor stage & & & 0.748 \\
\hline$|/| \mid$ & 38 & 18 & \\
\hline III/IV & 8 & 5 & \\
\hline Metastasis & & & 0.840 \\
\hline Mo & 35 & 18 & \\
\hline M1 & 11 & 5 & \\
\hline Size (longest diameter, cm) & & & 0.158 \\
\hline$<=7$ & 33 & 20 & \\
\hline$>7$ & 13 & 3 & \\
\hline Vital status (at followed-up) & & & $0.039 *$ \\
\hline Alive & 31 & 21 & \\
\hline Dead & 15 & 2 & \\
\hline
\end{tabular}

* P-value $<0.05$
GPD2 gene promoter sequences. One candidate site at 38 bp of GPD1 and two candidate sites at -288 bp and $665 \mathrm{bp}$ of GPD2 were predicted (Fig. 4D). To test whether HIF1 $\alpha$ binds at these sites to target GPD1 or GPD2, we performed a CUT\&RUN assay in RCC4 cells. The results demonstrated the direct association of HIF1 $\alpha$ with the GPD1 and GPD2 promoters (Fig. 4E and F). Furthermore, to verify whether the HBS within the GPD1 and GPD2 promoters functioned as hypoxia response elements (HRE), the fragment that encompassed the binding site of GPD1 at - 38 bp upstream of the firefly luciferase-coding region was inserted into the pGL4 plasmid. The GPD2 promoter region [700 bp before the transcription start site (TSS)] containing GPD2-HBS1 (-665 bp) and GPD2HBS2 (- $288 \mathrm{bp}$ ), as well as the GPD2 promoter sequence (300 bp before TSS) containing only GPD2-HBS2 (- 288 $\mathrm{bp})$, were both cloned upstream of the coding sequences for the firefly luciferase gene. Then, the luciferase reporter assay was performed and the results showed that GPD1HBS significantly increased luciferase activity as compared to the negative control group. Additionally, GPD2-HBS1 plus HBS2 induced a significant reduction in luciferase activity as compared to the GPD2-HBS2 group or negative control group in HEK293 cells (Fig. 4G).

GPD1 and HIF1a form a positive feedback loop to inhibit the expression of GPD2 and mitochondrial function HIF $1 \alpha$ was shown to negatively regulate the expression of GPD2, so we hypothesized that GPD1 modulated

Table 2 Univariate and multivariate analysis of different prognostic parameters in cCRCC patients

\begin{tabular}{lllllll}
\hline Variables & \multicolumn{3}{l}{ Univariate analysis } & \multicolumn{4}{l}{ Multivariate analysis } \\
\hline & $\mathrm{HR}$ & $\mathrm{Cl}$ & $\mathrm{P}$-value & $\mathrm{HR}$ & $\mathrm{Cl}$ & P-value \\
Age & 2.951 & $1.135-7.671$ & $0.026^{*}$ & 3.487 & $1.155-10.525$ & $0.027^{*}$ \\
Sex & 0.656 & $0.214-2.017$ & 0.462 & 0.329 & $0.095-1.140$ & 0.080 \\
T stage & 1.056 & $0.303-3.678$ & 0.932 & 0.543 & $0.131-2.247$ & 0.399 \\
Grade & 1.843 & $0.701-4.845$ & 0.215 & 4.198 & $0.854-20.638$ & 0.077 \\
Metastasis & 2.542 & $0.965-6.693$ & 0.059 & 2.957 & $0.969-9.024$ & 0.057 \\
Size & 2.261 & $0.834-6.130$ & 0.109 & 0.719 & $0.149-3.456$ & 0.680 \\
GPD1 & 0.213 & $0.049-0.930$ & $0.040^{*}$ & 0.124 & $0.018-0.868$ & $0.036^{*}$ \\
* P-value $<0.05$ & & & & &
\end{tabular}




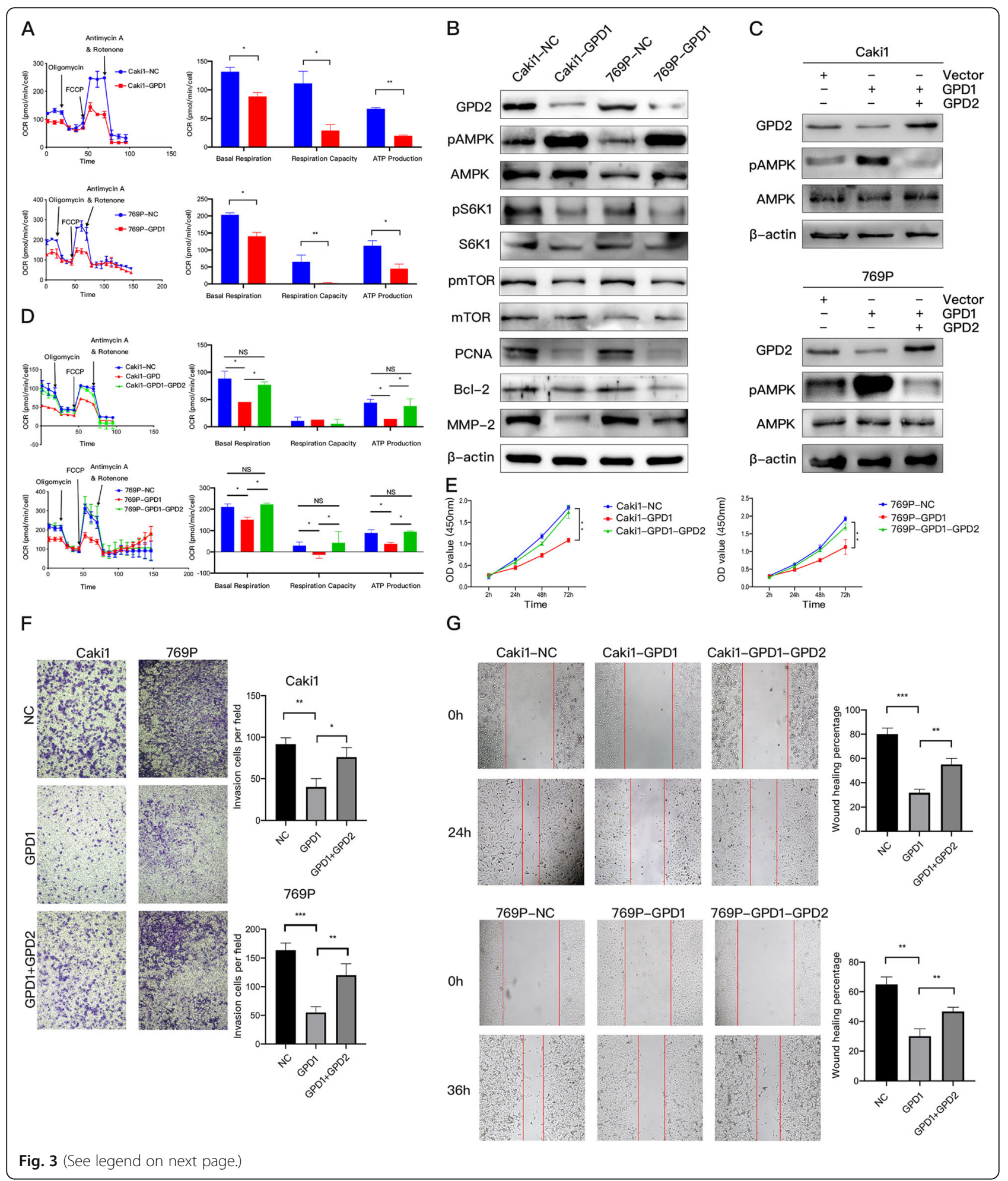


(See figure on previous page.)

Fig. 3 Overexpression of GPD1 inhibited mitochondrial function and induced AMPK phosphorylation by suppressing the expression of GPD2. (A) Oxygen consumption rate (OCR) of Caki1 and 769P cells with GPD1 overexpression. Baseline respiration, spare respiratory capacity, and ATP production in Caki1 and 769P cells with GPD1 overexpression. (B) Western blotting of indicated proteins in Caki1-NC, Caki1-GPD1, 769P -NC, and 769P-GPD1 cells. (C) Western blotting of GPD2, PAMPK, and AMPK in Caki1-GPD1 and 769P-GPD1 cells with GPD2 restoration and a negative control. (D) The OCR in Caki1-GPD1 and 769P-GPD1 cells with GPD2 restoration and a negative control. Baseline respiration, spare respiratory capacity, and ATP production in Caki1-GPD1 and 769P-GPD1 cells with GPD2 restoration and a negative control are shown. (E) Cell proliferation in Caki1-GPD1 and 769P-GPD1 cells with GPD2 restoration and a negative control as examined by CCK-8 assays. (F-G) Cell migration and invasion in Caki1-GPD1 and 769P-GPD1 cells with GPD2 restoration and a negative control as examined by wound healing and Transwell assays. Red lines denote the margins of the wound. Data are shown as the mean + SD $(n=3)$. Statistical analysis was performed using an unpaired Student's $t$ test with a two-tailed distribution (NS, $p>0.05,{ }^{*} p<0.05,{ }^{* *} p<0.01,{ }^{* * *} p<0.001$ ). Abbreviations: GPD1, glycerol-3-phosphate dehydrogenase 1; GPD2, glycerol-3-phosphate dehydrogenase 2; CCK-8, cell counting kit

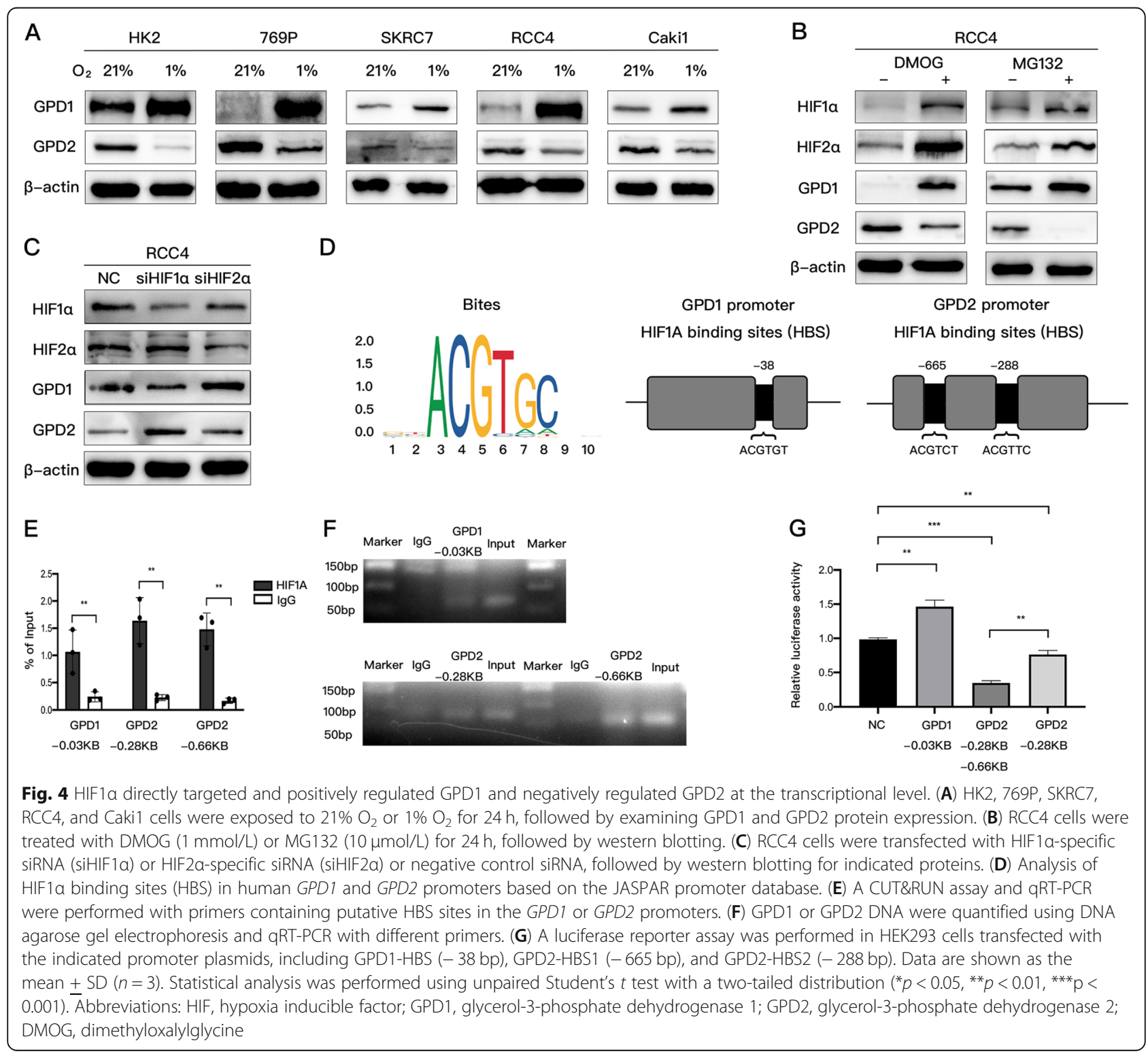




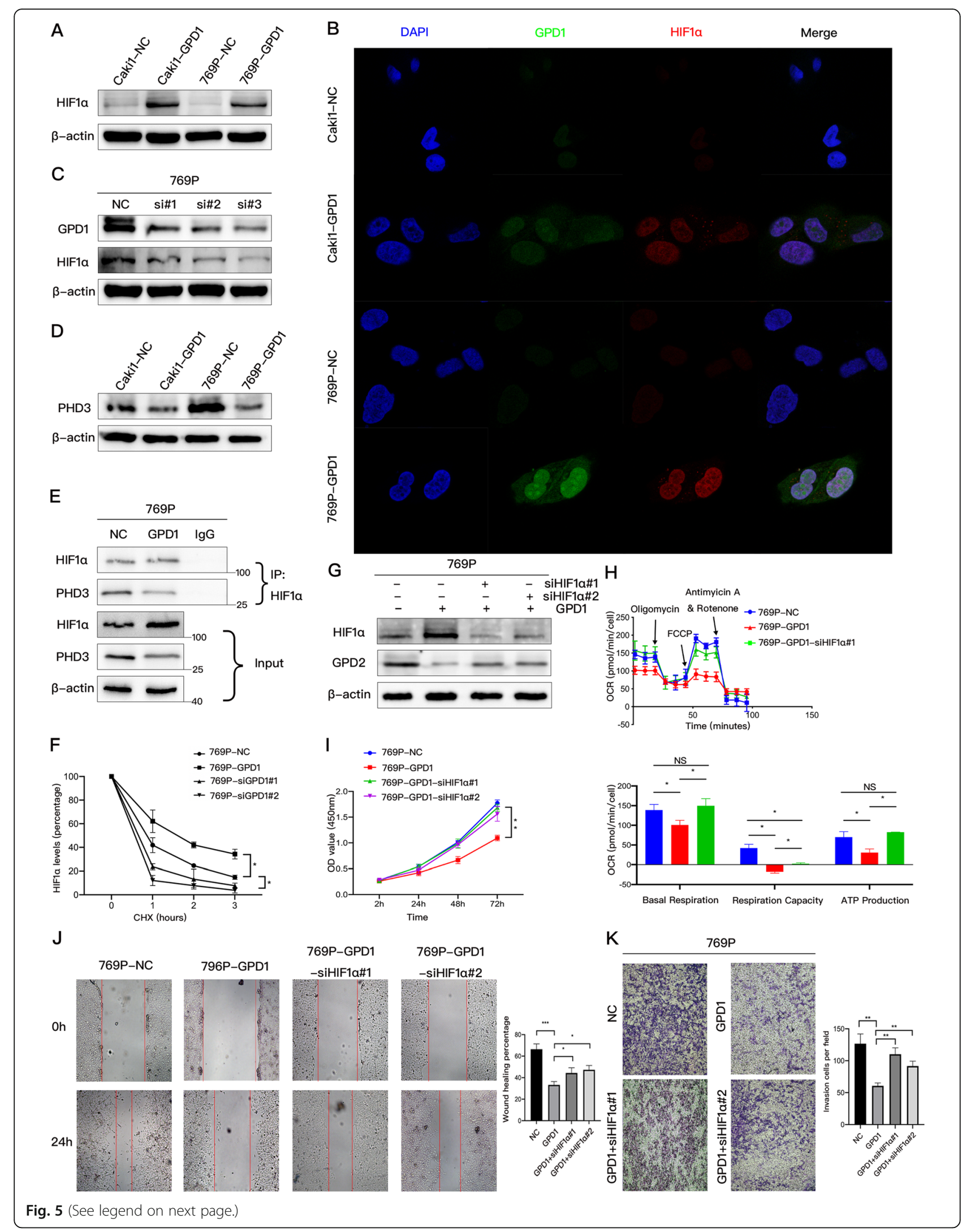




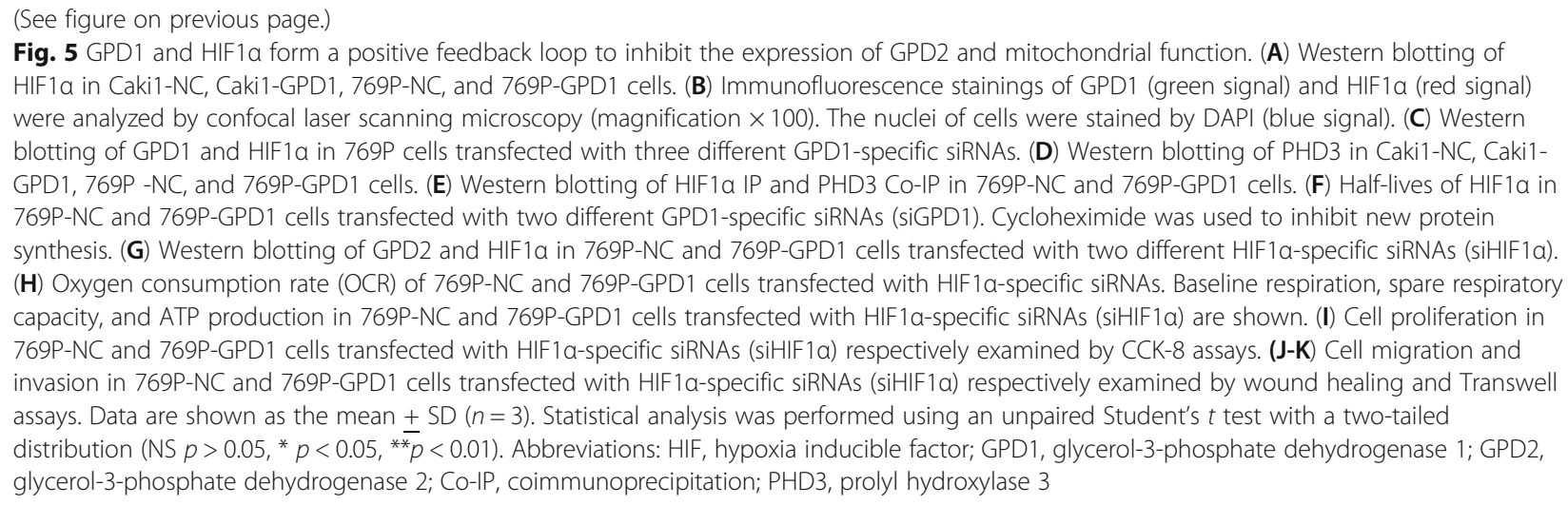

HIF1 $\alpha$ stability to inhibit the expression of GPD2. To evaluate this hypothesis, we overexpressed GPD1 in Caki1 and 769P cells, which resulted in a significant increase in HIF1 $\alpha$ protein (Fig. 5A). Immunofluorescence indicated that the expression of HIF $1 \alpha$ was enhanced by the overexpression of GPD1, while HIF1 $\alpha$ was expressed both in the nucleus and cytoplasm (Fig. 5B). Further, we knocked down the expression of GPD1 using three different kinds of siRNA, which resulted in a decrease of HIF1 $\alpha$ in 769P cells (Fig. 5C). However, overexpression of GPD1 was unable to affect the mRNA expression of $H I F 1 \alpha$ (Fig. S5A), indicating that GPD1 regulated the expression of HIF1 $\alpha$ at the protein level, but not the mRNA level.

We previously performed RNA-seq on 786O-NC and 786O-GPD1 cells [14], and the results suggested that overexpression of GPD1 induces the downregulation of PHD3 at the mRNA level. We further confirmed this downregulation at the protein level by western blotting (Fig. 5D). PHD3 is one of the three HIF prolyl hydroxylases, which includes PHD1-3. We then inferred that GPD1 reduces the degradation of HIF1 $\alpha$ via inhibiting the expression of PHD3, which also explained why GPD1 overexpression did not change the mRNA level of HIF1 $\alpha$. Furthermore, a Co-IP assay was performed with anti-HIF1 $\alpha$ and anti-PHD3 antibodies to confirm that overexpression of GPD1 decreased the interaction of HIF1 $\alpha$ with PHD3 in HEK293 cells (Fig. 5E). To investigate whether GPD1 can prolong the half-life of endogenous HIF1 $\alpha$, we overexpressed or knocked down GPD1 in $769 \mathrm{P}$ cells and treated cells with a protein synthesis inhibitor, cycloheximide, for different time periods. As shown in Figs. 5F and S5B, GPD1 can significantly affect the decay of HIF1 $\alpha$ protein and prolong the half-life of HIF1 $\alpha$.

Additionally, we knocked down the expression of HIF1 $\alpha$ with two different kinds of siRNA in 769P-GPD1 cells, which led to the restoration of GPD2 expression (Fig. 5G). To further confirm this observation, we performed a seahorse assay using 769P-GPD1 cells transfected with siHIF1 $\alpha$, and the results showed that mitochondrial function in these cells was restored (Fig. $5 \mathrm{H})$. Then CCK-8, wound healing and Transwell assays were performed, which indicated that knockdown of HIF $1 \alpha$ significantly restored the proliferation, migration, and invasion abilities of 769P-GPD1 cells (Fig. 5I, J, and K).

\section{Overexpression of GPD1 inhibited lipid metabolism in cCRCC cells}

Based on the RNA-seq data of the 786O-NC and 786OGPD1 cells [14], KEGG and GO functional enrichment analyses were conducted to uncover the gene function and biological pathways of DEGs. From these analyses, we found that GPD1 mainly exerted its function via lipid metabolism pathways, such as fatty acid metabolism and cholesterol metabolism (Fig. 6A). Furthermore, Gene Set Enrichment Analysis (GSEA) was performed on these RNA-seq data, and the results suggested that the gene sets that positively correlated with sterol biosynthesis, fatty acyl-CoA metabolism, and sterol metabolism were less enriched in the group overexpressing GPD1 (FDR < $25 \%$ and $p<0.05$; Fig. 6B). In addition, the gene sets that positively correlated with glycerophospholipid metabolism were more enriched in the group overexpressing GPD1 (Fig. S6A). As shown in Fig. 6C, several key proteins for fatty acid and lipid metabolism including acetyl-CoA synthetase (AceCS1), acyl-CoA synthetase (ACSL1), ATP-citrate lyase (ATP-CL), Lipin1, acetylCoA carboxylase (ACC1), and fatty acid synthase (FAS), were all downregulated in Caki1 and 769P cells with GPD1 overexpression. These results indicated that overexpression of GPD1 inhibited lipid metabolism, and to confirm this, a free fatty acid assay and Oil Red O staining were performed to assess the levels of free fatty acids and the accumulation of adipocyte lipid droplets, respectively. We found that the two cell lines overexpressing GPD1 showed lower levels of free fatty acids and 


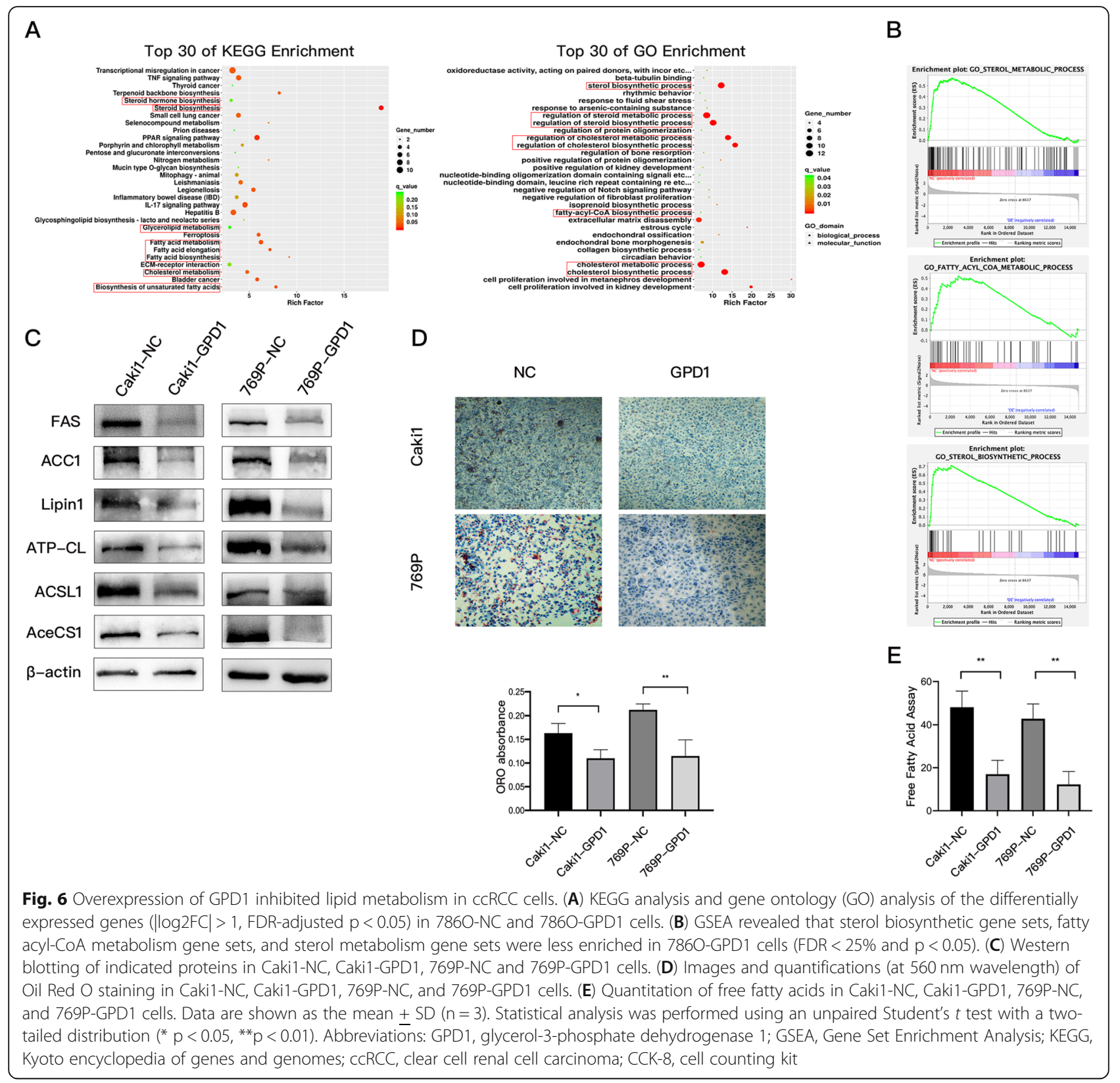

developed lipid droplets, displaying a dramatic reduction as compared to the negative control cells under the same conditions (Fig. 6D and E). However, overexpression of GPD1 significantly increased total phospholipid levels according to a phospholipid assay (Fig. S6B).

\section{Discussion}

ccRCC is considered to be a metabolic disease characterized by the accumulation of lipids and glycogen, which contributes to a wide variety of metabolic defects and perturbations that occur as a result of unique genetic characteristics [27]. Thus far, there have been many studies on hypoxic pathways and metabolic pathways in
ccRCC. One study demonstrated that HIF $2 \alpha$ induces hypoxia-dependent cell proliferation by activating CMYC activity [28]. Qiu et al. reported that HIF2 $\alpha$ dependent PLIN2 expression promotes lipid storage, in addition to the proliferation and progression of xenograft tumors [29]. Another study found that carnitine palmitoyltransferase $1 \mathrm{~A}(\mathrm{CPT} 1 \mathrm{~A})$, which is negatively and directly regulated by HIF $1 \alpha$ and HIF $2 \alpha$ at the transcriptional level, reduces the transportation of fatty acids into the mitochondria and forces the formation of lipid droplets from fatty acids for storage [2]. In this study, we discovered that HIF $1 \alpha$ can positively regulate GPD1 at the transcriptional level to suppress tumor progression 
via inhibiting mitochondrial function and lipid metabolism. Our data revealed a new mechanism by which the hypoxic and lipid metabolism pathways are involved in the progression of ccRCC.

Madiraju et al. found that metformin can inhibit gluconeogenesis by inhibiting GPD2 to induce AMPK phosphorylation and proposed that GPD2 may be a target for tumor therapy [12, 30]. Subsequent studies support this view in tumors, including thyroid cancer [13], prostate cancer [31], and lung cancer [14]. We are the first to propose that in ccRCC, GPD1 can suppress the expression of GPD2, thereby inhibiting mitochondrial function and inducing AMPK phosphorylation. We confirmed that HIF $1 \alpha$ can negatively regulate the expression of GPD2 at the transcriptional level, thereby inhibiting tumor progression, which is new mechanism-of-action for HIF1 $\alpha$ as a tumor suppressor. In addition, GPD1 can also stabilize the expression of HIF $1 \alpha$ by inhibiting the expression of PHD3 and reducing the degradation of HIF1 $\alpha$. Therefore, GPD1 can form a positive feedback loop with HIF1 $\alpha$ to regulate GPD2 expression and mitochondrial function (Fig. 7), but the specific mechanism by which GPD1 affects PHD3 needs further study.

Bioinformatic analysis showed that GPD1 may play an essential role in the lipid metabolism pathway. Another study found that GPD1 mutations can lead to hypertriglyceridemia and a fatty liver in infants [32]. Our RNA-seq analysis and experiments also confirmed that overexpression of GPD1 led to the significant inhibition of lipid metabolism pathways. However, glycerol-3phosphate (G3P), a product catalyzed by GPD1, should increase after the overexpression of GPD1, which provides more substrates for the synthesis of triglyceride. We speculate that increased G3P is more inclined to produce glycerophospholipids, which leads to a decrease in the production of lipids including triglycerides and fatty acids. Some research has shown that ccRCC patients have lower levels of phospholipids, and that the phosphatidylethanolamine (PE) synthesis pathway is suppressed in cancerous tissues, which is associated with cell proliferation $[3,8]$. The results of GSEA and phospholipid assays (Figs. S6A and S6B) support this speculation, but the mechanism needs more research. Our previous studies have found that in prostate cancer and lung cancer, GPD1 can enhance the antitumor effect of metformin, with G3P playing a major role in inhibiting tumor proliferation [14]. Thus, we believe that G3P may have an instantaneous effect, because exogenous G3P was increased in a short period of time, while GPD2 inhibition was a longterm effect, as the activation of the hypoxic pathway required a longer process.

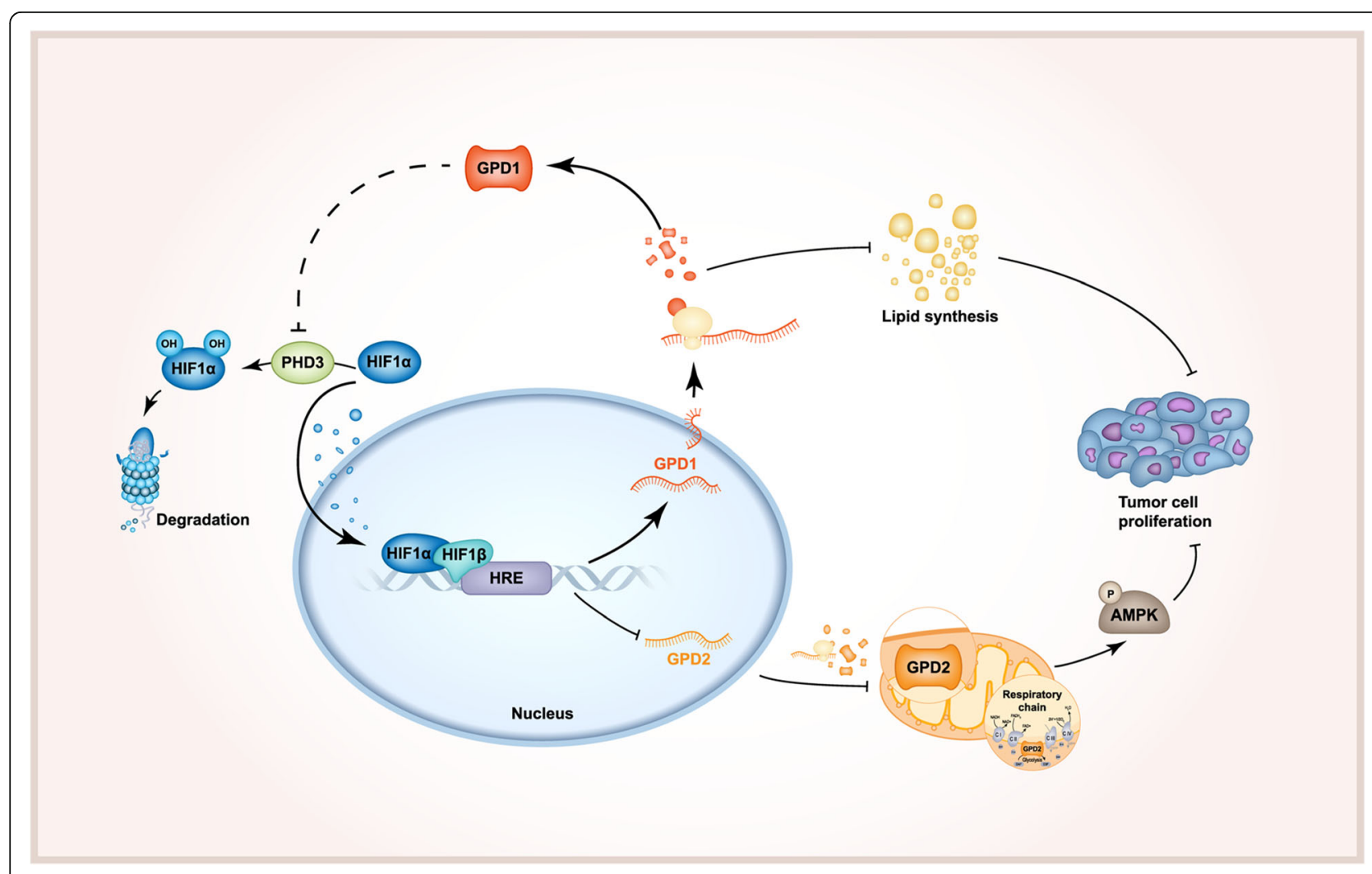

Fig. 7 Schematic diagram of the HIF1a-GPD1 feedforward loop 
Clinically, detecting GPD1 expression can be used for the early diagnosis of ccRCC and provide guidance for the prognosis of the disease. In addition, based on the role of GPD1 in tumor proliferation and migration, it is possible to find a drug that binds the enzyme receptor at its binding site and promotes the expression of GPD1, which could provide a novel therapy for ccRCC, but this requires further study.

\section{Conclusions}

In summary, our study confirmed that GPD1 forms a positive feedback loop with HIF1 $\alpha$ to decrease the expression of GPD2, a key target involved in mitochondrial oxidative phosphorylation. This results in the inhibition of mitochondrial function, induction of AMPK phosphorylation, and suppression of lipid metabolism (Fig. 7). This study revealed a new mechanism underlying the tumor suppression ability of HIF $1 \alpha$ and provides insights into the role of hypoxia and lipid metabolism in tumor progression. The results of this study have the potential to be used in the development of novel therapies for ccRCC.

\section{Abbreviations \\ ccRCC: clear cell renal cell carcinoma; GPD1: Glycerol-3-phosphate dehydrogenase 1; HIF: Hypoxia inducible factor; IHC: Immunohistochemical; TMA: Tissue microarray; qRT-PCR: quantitative real-time PCR analysis; \\ TCGA: The Cancer Genome Atlas; CCK-8: Cell counting kit}

\section{Supplementary Information}

The online version contains supplementary material available at https://doi. org/10.1186/s13046-021-01996-6.

\section{Additional file 1.}

\section{Acknowledgments}

Not applicable.

\section{Authors' contributions}

RL, YF-F, YL-D, ZH-Z, and XJ-Z acquired the data. RL, YF-F, JH-Y, and ZD-C analyzed and interpreted the data. RL, YF-F, and JM-L conceived and designed research. YK-L and HZ contributed GPD1 constructs. YL, ZD-H, and YJ-Z have provided primary tumor cells and microdissected human tumor vs nontumor samples. RL, QL-X, YX-L, CL-W and WD-Z drafted the manuscript. CT-H, $Y X-L, C L-W$. and WD-Z handled the funding and supervision. The author(s) read and approved the final manuscript.

\section{Funding}

This work was supported by grants from National Natural Science Foundation of China (82072813, 81874099, 82003271); the Fundamental Research Funds for the Central Universities (2018MS18); Natural Science Foundation of Guangdong Province (2020A1515010473, 2018A030313668, 2020A1515110792); Guangzhou Municipal Science and Technology Project (201803040001); The Science Foundation of Guangzhou First People's Hospital (Q2019020, Q2019002).

\section{Availability of data and materials}

The datasets used and/or analyzed during this study are available from the corresponding author on reasonable request.

\section{Declarations}

\section{Ethics approval and consent to participate}

All human tissue samples were approved by the Human Ethics Committee of the Public Health Department of the People's Republic of China and the Massachusetts General Hospital (MGH). All animal experiments were performed in accordance with the guidelines of the Institute for Laboratory Animal Research and the National Cancer Center Research Institute.

\section{Consent for publication}

Not applicable.

\section{Competing interests}

The authors declare that they have no possible conflicts of interest.

\section{Author details}

${ }^{1}$ Guangdong Provincial Institute of Nephrology, Nanfang Hospital, Southern Medical University, Guangzhou 510515, China. ${ }^{2}$ Department of Urology, Guangdong Key Laboratory of Clinical Molecular Medicine and Diagnostics, Guangzhou First People's Hospital, School of Medicine, South China University of Technology, Guangzhou 510180, China. ${ }^{3}$ Urology Key Laboratory of Guangdong Province, The First Affiliated Hospital of Guangzhou Medical University, Guangzhou Medical University, Guangzhou 510230, China. ${ }^{4}$ Department of Urology, the Fifth Affiliated Hospital of Guangzhou Medical University, Guangzhou 510700, China. ${ }^{5}$ Department of Urology, Affiliated Foshan Hospital of Southern Medical University, Southern Medical University, Foshan 528000, China. ${ }^{6}$ Macau Institute of Systems Engineering, Macau University of Science and Technology, Avenida Wai Long, Taipa, Macau 999078, China. ${ }^{7}$ Departments of Urology, Massachusetts General Hospital and Harvard Medical School, Boston, MA 02114, USA. ${ }^{8}$ Department of Pathology, Massachusetts General Hospital and Harvard Medical School, Boston, MA 02114, USA.

Received: 26 January 2021 Accepted: 27 May 2021

Published online: 07 June 2021

\section{References}

1. Moch H, Cubilla AL, Humphrey PA, Reuter VE, Ulbright TM. The 2016 WHO classification of Tumours of the urinary system and male genital organs-part a: renal, penile, and testicular Tumours. Eur Urol. 2016;70(1):93-105. https:// doi.org/10.1016/j.eururo.2016.02.029.

2. Du W, et al. HIF drives lipid deposition and cancer in ccRCC via repression of fatty acid metabolism. Nat Commun. 2017;8(1):1769. https://doi.org/10.1 038/s41467-017-01965-8

3. Hakimi AA, Reznik E, Lee CH, Creighton CJ, Brannon AR, Luna A, et al. An integrated metabolic atlas of clear cell renal cell carcinoma. Cancer Cell. 2016;29(1):104-16. https://doi.org/10.1016/j.ccell.2015.12.004.

4. Shen C, Beroukhim R, Schumacher SE, Zhou J, Chang M, Signoretti S, et al. Genetic and functional studies implicate HIF1alpha as a 14q kidney cancer suppressor gene. Cancer Discov. 2011;1(3):222-35. https://doi.org/10.11 58/2159-8290.CD-11-0098.

5. Hoefflin R, Harlander S, Schäfer S, Metzger P, Kuo F, Schönenberger D, et al. HIF-1alpha and HIF-2alpha differently regulate tumour development and inflammation of clear cell renal cell carcinoma in mice. Nat Commun. 2020; 11(1):4111. https://doi.org/10.1038/s41467-020-17873-3.

6. Melendez-Rodriguez F, et al. HIF1alpha suppresses tumor cell proliferation through inhibition of aspartate biosynthesis. Cell Rep. 2019;26(9):2257-65 e4. https://doi.org/10.1016/j.celrep.2019.01.106.

7. Schodel J, et al. Hypoxia, hypoxia-inducible transcription factors, and renal Cancer. Eur Urol. 2016;69(4):646-57. https://doi.org/10.1016/.jeururo.2015.08.007.

8. Saito K, Arai E, Maekawa K, Ishikawa M, Fujimoto H, Taguchi R, et al. Lipidomic signatures and associated transcriptomic profiles of clear cell renal cell carcinoma. Sci Rep. 2016;6(1):28932. https://doi.org/10.1038/srep28932.

9. Swierczynski J, Zabrocka L, Goyke E, Raczynska S, Adamonis W, Sledzinski Z. Enhanced glycerol 3-phosphate dehydrogenase activity in adipose tissue of obese humans. Mol Cell Biochem. 2003;254(1-2):55-9. https://doi.org/10.1 023/A:1027332523114.

10. Eto K, Kadowaki T. Role of the NADH shuttle system in glucose-induced insulin secretion. Nihon Rinsho. 1999;57(3):503-14.

11. Mracek T, Drahota Z, Houstek J. The function and the role of the mitochondrial glycerol-3-phosphate dehydrogenase in mammalian tissues. 
Biochim Biophys Acta. 2013;1827(3):401-10. https://doi.org/10.1016/j.bba bio.2012.11.014.

12. Madiraju AK, Erion DM, Rahimi Y, Zhang XM, Braddock DT, Albright RA, et al. Metformin suppresses gluconeogenesis by inhibiting mitochondrial glycerophosphate dehydrogenase. Nature. 2014;510(7506):542-6. https://doi. org/10.1038/nature13270

13. Thakur S, Daley B, Gaskins K, Vasko W, Boufraqech M, Patel D, et al. Metformin targets mitochondrial Glycerophosphate dehydrogenase to control rate of oxidative phosphorylation and growth of thyroid Cancer in vitro and in vivo. Clin Cancer Res. 2018;24(16):4030-43. https://doi.org/1 0.1158/1078-0432.CCR-17-3167.

14. Xie J, Ye J, Cai Z, Luo Y, Zhu X, Deng Y, et al. GPD1 enhances the anticancer effects of metformin by synergistically increasing Total cellular Glycerol-3-phosphate. Cancer Res. 2020;80(11):2150-62. https:/doi.org/10.1158/0008-5472.CAN-19-2852.

15. Balabanov S, Zimmermann U, Protzel C, Scharf C, Klebingat KJ, Walther R. Tumour-related enzyme alterations in the clear cell type of human renal cell carcinoma identified by two-dimensional gel electrophoresis. Eur J Biochem. 2001;268(22):5977-80. https://doi.org/10.1046/j.0014-2956.2001.02546.x.

16. Cai Z, Deng Y, Ye J, Zhuo Y, Liu Z, Liang Y, et al. Aberrant expression of citrate synthase is linked to disease progression and clinical outcome in prostate Cancer. Cancer Manag Res. 2020;12:6149-63. https://doi.org/10.2147/CMAR.S255817.

17. Simon N, Friedman J, Hastie T, Tibshirani R. Regularization paths for Cox's proportional hazards model via coordinate descent. J Stat Softw. 2011;39(5): 1-13. https://doi.org/10.18637/jss.v039.105.

18. Zhang Y, Wang J, Liu X. LRRC19-A Bridge between Selenium Adjuvant Therapy and Renal Clear Cell Carcinoma: A Study Based on Datamining. Genes (Basel). 2020;11(4):440. Published 2020 Apr 17. https://doi.org/10.33 90/genes 11040440.

19. Pflueger D, Mittmann C, Dehler S, Rubin MA, Moch H, Schraml P. Functional characterization of BC039389-GATM and KLK4-KRSP1 chimeric read-through transcripts which are up-regulated in renal cell cancer. BMC Genomics. 2015;16(1):247. https://doi.org/10.1186/s12864-015-1446-z.

20. Morrissey JJ, Kharasch ED. The specificity of urinary aquaporin 1 and perilipin 2 to screen for renal cell carcinoma. J Urol. 2013;189(5):1913-20. https://doi.org/10.1016/j.juro.2012.11.034.

21. Hisazumi H, Nakajima K, Nishino A, Misaki T, Migita S. Pre- and postoperative changes of serum proteins in renal cancer patients. Hinyokika Kiyo. 1985;31(9):1519-23.

22. Liu Y, Wang H, Ni B, Zhang J, Li S, Huang Y, et al. Loss of KCNJ15 expression promotes malignant phenotypes and correlates with poor prognosis in renal carcinoma. Cancer Manag Res. 2019;11:1211-20. https://doi.org/10.214 7/CMAR.S184368.

23. Mei J, Hu K, Peng X, Wang H, Liu C. Decreased expression of SLC16A12 mRNA predicts poor prognosis of patients with clear cell renal cell carcinoma. Medicine (Baltimore). 2019;98(30):e16624. https://doi.org/10.1 097/MD.0000000000016624.

24. Takenawa J, Kaneko Y, Kishishita M, Higashitsuji H, Nishiyama H, Terachi T, et al. Transcript levels of aquaporin 1 and carbonic anhydrase IV as predictive indicators for prognosis of renal cell carcinoma patients after nephrectomy. Int J Cancer. 1998;79(1):1-7. https://doi.org/10.1002/(SICI)1 097-0215(19980220)79:1<1::AID-IJC1>3.0.CO;2-5.

25. Chen ZF, Xiao YJ, Huang ZH, Chen T, Zhao SC, Jiang YD, et al. Quantitative and comparative proteomics analysis in clear cell renal cell carcinoma and adjacent noncancerous tissues by 2-D DIGE. Nan Fang Yi Ke Da Xue Xue Bao. 2017;37(11):1517-22.

26. Galgano MT, Hampton GM, Frierson HF Jr. Comprehensive analysis of HE4 expression in normal and malignant human tissues. Mod Pathol. 2006;19(6): 847-53. https://doi.org/10.1038/modpathol.3800612.

27. Rathmell WK, Rathmell JC, Linehan WM. Metabolic Pathways in Kidney Cancer: Current Therapies and Future Directions. J Clin Oncol. 2018 JCO2018792309. https://doi.org/10.1200/JCO.2018.79.2309. Epub ahead of print.

28. Gordan JD, Bertout JA, Hu CJ, Diehl JA, Simon MC. HIF-2alpha promotes hypoxic cell proliferation by enhancing c-myc transcriptional activity. Cancer Cell. 2007;1 1(4):335-47. https://doi.org/10.1016/j.ccr.2007.02.006.

29. Oiu B, Ackerman D, Sanchez DJ, Li B, Ochocki JD, Grazioli A, et al. HIF2alphadependent lipid storage promotes endoplasmic reticulum homeostasis in clear-cell renal cell carcinoma. Cancer Discov. 2015;5(6):652-67. https://doi. org/10.1158/2159-8290.CD-14-1507.
30. Singh G. Mitochondrial FAD-linked Glycerol-3-phosphate dehydrogenase: a target for Cancer therapeutics. Pharmaceuticals (Basel). 2014;7(2):192-206. https://doi.org/10.3390/ph7020192.

31. Pecinova A, et al. Role of Mitochondrial Glycerol-3-Phosphate Dehydrogenase in Metabolic Adaptations of Prostate Cancer. Cells. 2020; 9(8):1764. Published 2020 Jul 23. doi:10.3390/cells9081764

32. Basel-Vanagaite L, Zevit N, Zahav AH, Guo L, Parathath S, Pasmanik-Chor M, et al. Transient infantile hypertriglyceridemia, fatty liver, and hepatic fibrosis caused by mutated GPD1, encoding glycerol-3-phosphate dehydrogenase 1. Am J Hum Genet. 2012;90(1):49-60. https//doi.org/10.1016/j.ajhg.2011.11.028.

\section{Publisher's Note}

Springer Nature remains neutral with regard to jurisdictional claims in published maps and institutional affiliations.

\section{Ready to submit your research? Choose BMC and benefit from:}

- fast, convenient online submission

- thorough peer review by experienced researchers in your field

- rapid publication on acceptance

- support for research data, including large and complex data types

- gold Open Access which fosters wider collaboration and increased citations

- maximum visibility for your research: over $100 \mathrm{M}$ website views per year

At BMC, research is always in progress.

Learn more biomedcentral.com/submissions 